\title{
4-Cycle Decompositions of Graphs
}

\author{
Teresa Sousa \\ Departamento de Matemática and Centro de Matemática e Aplicações, \\ Faculdade de Ciências e Tecnologia, Universidade Nova de Lisboa, Lisbon, Portugal \\ Email: tmjs@fct.unl.pt
}

Received May 10, 2012; revised June 3, 2012; accepted August 6, 2012

\begin{abstract}
In this paper we consider the problem of finding the smallest number $\phi$ such that any graph $G$ of order $n$ admits a decomposition into edge disjoint copies of $C_{4}$ and single edges with at most $\phi$ elements. We solve this problem for $n$ sufficiently large.
\end{abstract}

Keywords: Graph Decomposition; 4-Cycle Packing; Graph Packing

\section{Introduction}

All graphs in this paper are finite, undirected and simple. For notation and terminology not discussed here the reader is referred to [1].

Given two graphs $G$ and $H$, an $H$-decomposition of $G$ is a partition of the edge set of $G$ such that each part is either a single edge or forms an $H$-subgraph, i.e., a graph isomorphic to $H$. We allow partitions only, that is, every edge of $G$ appears in precisely one part. Let $\phi_{H}(G)$ be the smallest possible number of parts in an $H$-decomposition of $G$. For non-empty $H$, let

$p_{H}(G)$ be the maximum number of pairwise edgedisjoint $H$-subgraph that can be packed into $G$ and $e(G)$ the number of edges in $G$. It is easy to see that

$$
\phi_{H}(G)=e(G)-p_{H}(G)(e(H)-1) .
$$

Here we study the function

$$
\phi_{H}(n)=\max \left\{\phi_{H}(G) \mid v(G)=n\right\},
$$

which is the smallest number, such that, any graph $G$ of order $n$ admits an $H$-decomposition with at most $\phi_{H}(n)$ elements.

The function $\phi_{H}(n)$ was first studied by Erdös, Goodman and Pósa [2], who proved that $\phi_{K_{3}}(n)=t_{2}(n)$, where $K_{r}$ denotes the complete graph (clique) of order $r$ and $t_{r}(n)$ is the maximum size of an $r$-partite graph on $n$ vertices. A decade later, this result was extended by Bollobás [3], who proved that

$$
\phi_{K_{r}}(n)=t_{r-1}(n), \text { for all } n \geq r \geq 3 .
$$

Recently, Pikhurko and Sousa [4] studied $\phi_{H}(n)$ for arbitrary graphs $H$.

Theorem 1.1. (See Theorem 1.1 from [4]) Let $H$ be any fixed graph of chromatic number $r \geq 3$. Then,

$$
\phi_{H}(n)=t_{r-1}(n)+o\left(n^{2}\right) .
$$

Let ex $(n, H)$ denote the maximum number of edges in a graph of order $n$, that does not contain $H$ as a subgraph. Recall that ex $\left(n, K_{r}\right)=t_{r-1}(n)$. Pikhurko and Sousa [4] also made the following conjecture.

Conjecture 1. For any graph $H$ with chromatic number at least 3 , there is $n_{0}=n_{0}(H)$ such that $\phi_{H}(n)=\operatorname{ex}(n, H)$, for all $n \geq n_{0}$.

The exact value of the function $\phi_{H}(n)$ is far from being known. Sousa determined it for a few special edgecritical graphs, namely for clique-extensions of order $r \geq 4 \quad(n \geq r)$ [5] and the cycles of length $5(n \geq 6)$ and $7(n \geq 10)$ [6,7]. Later, Özkahya and Person [8] determined it for all edge-critical graphs with chromatic number $r \geq 3$ and $n$ sufficiently large. They proved the following result.

Theorem 1.2. ([8]) Let $H$ be any edge-critical graph with chromatic number $r \geq 3$. Then, there exists $n_{0}$ such that $\phi_{H}(n)=\operatorname{ex}(n, H)$, for all $n \geq n_{0}$. Moreover, the only graph attaining $\phi_{H}(n)$ is the Turán graph $T_{r-1}(n)$.

Recently, Allen, Böttcher and Person [9] improved the error term obtained by Pikhurko and Sousa in Theorem 1.1 .

The case when $H$ is a bipartite graph has been less studied. Pikhurko and Sousa [4] determined $\phi_{H}(n)$ for any fixed bipartite graph with an $O(1)$ additive error. For a non-empty graph $H$, let $\operatorname{gcd}(H)$ denote the greatest common divisor of the degrees of $H$. For example, $\operatorname{gcd}\left(K_{6,4}\right)=2$ while for any tree $T$ with at least 2 vertices we have $\operatorname{gcd}(T)=1$. They proved the following result. 
Theorem 1.3. (See Theorem 1.3 from [4]) Let $H$ be a bipartite graph with $m$ edges and let $d=\operatorname{gcd}(H)$. Then there is $n_{0}=n_{0}(H)$ such that for all $n \geq n_{0}$ the following statements hold.

(1) If $d=1$, then $\phi_{H}(n)=\left\lfloor\frac{n(n-1)}{2 m}\right\rfloor+C$, where $C=m-1$ or $C=m-2$.

(2) If $d \geq 2$, then

$\phi_{H}(n)=\frac{n d}{2 m}\left(\left\lfloor\frac{n}{d}\right\rfloor-1\right)+\frac{1}{2} n(d-1)+O(1)$.

Moreover, there is a procedure running in polynomial in $\log n$ time which determines $\phi_{H}(n)$ and describes a family $\mathcal{D}$ of $n$-sequences such that a graph $G$ of order $n$ satisfies $\phi_{H}(G)=\phi_{H}(n)$ if and only if the degree sequence of $G$ belongs to $\mathcal{D}$. (It will be the case that $|\mathcal{D}|=O(1)$ and each sequence in $\mathcal{D}$ has $n-O(1)$ equal entries, so $\mathcal{D}$ can be described using $O(\log n)$ bits.

Here we will determine the exact value of $\phi_{C_{4}}(n)$ for $n$ sufficiently large.

Theorem 1.4. There is $n_{0}=n_{0}\left(C_{4}\right)$ such that for all $n \geq n_{0}$ the following statements hold.

(1) If $n$ is even then $\phi_{C_{4}}(n)=\frac{n^{2}}{8}+\frac{n}{4}+1$.

(2) If $n \equiv 1(\bmod 8)$ then $\phi_{C_{4}}(n)=\frac{n^{2}}{8}+\frac{n}{8}+\frac{14}{8}$.

(3) If $n \equiv 3(\bmod 8)$ then $\phi_{C_{4}}(n)=\frac{n^{2}}{8}+\frac{n}{8}+\frac{3}{2}$.

(4) If $n \equiv 5(\bmod 8)$ then $\phi_{C_{4}}(n)=\frac{n^{2}}{8}+\frac{n}{8}+\frac{10}{8}$.

(5) If $n \equiv 7(\bmod 8)$ then $\phi_{C_{4}}(n)=\frac{n^{2}}{8}+\frac{n}{8}+2$.

\section{Proof of Theorem 1.4}

In this section we will prove Theorem 1.4, but first we need to introduce the tools. We start with the following easy result about $H$-decompositions.

Lemma 5. (Lemma 1.3) For any non-empty graph $H$ with $m$ edges and any integer $n$, we have

$$
\phi_{H}(n) \leq \frac{1}{m}\left(\begin{array}{l}
n \\
2
\end{array}\right)+\frac{m-1}{m} \operatorname{ex}(n, H) .
$$

In particular, if $H$ is a fixed bipartite graph with $m$ edges and $n \rightarrow \infty$, then

$$
\phi_{H}(n)=\left(\frac{1}{m}+O(1)\right)\left(\begin{array}{l}
n \\
2
\end{array}\right) .
$$

The following result is the well known Erdös-Gallai theorem that gives a necessary and sufficient condition for a finite sequence to be the degree sequence of a simple graph.

Theorem 2.6. (Erdős-Gallai Theorem [10]) Let $0 \leq d_{1} \leq \cdots \leq d_{n}$ be a sequence of integers. There is a graph with degree sequence $d_{1}, \cdots, d_{n}$ if and only if

(1) $d_{1}+\cdots+d_{n}$ is even;

(2) for each $1 \leq k \leq n$

$$
\sum_{i=n-k+1}^{n} d_{i} \leq k(k-1)+\sum_{i=1}^{n-k} \min \left\{d_{i}, k\right\} .
$$

The following results appearing in Alon, Caro and Yuster [11, Theorem 1.1, Corollary 3.4, Lemma 3.5] which follow with some extra work from the powerful decomposition theorem of Gustavsson [12] are essential to the proof of Theorem 1.4.

Lemma 2.7. For any non-empty graph $H$ with $m$ edges, there are $\gamma>0$ and $N_{0}$ such that the following holds. Let $d=\operatorname{gcd}(H)$. Let $G$ be a graph of order $n \geq N_{0}$ and of minimum degree $\delta(G) \geq(1-\gamma) n$.

If $d=1$, then

$$
p_{H}(G)=\left\lfloor\frac{e(G)}{m}\right\rfloor .
$$

If $d \geq 2$, let $\alpha_{u}=d\left\lfloor\frac{\operatorname{deg}(u)}{d}\right\rfloor$ for $u \in V(G)$ and let $X$ consist of all vertices whose degree is not divisible by $d$. If $|X| \geq \frac{n}{10 d^{3}}$, then

$$
p_{H}(G)=\left\lfloor\frac{1}{2 m} \sum_{u \in V(G)} \alpha_{u}\right\rfloor .
$$

$$
\begin{aligned}
& \text { If }|X|<\frac{n}{10 d^{3}} \text {, then } \\
& \qquad p_{H}(G) \geq \frac{1}{m}\left(e(G)-\frac{n}{5 d^{2}}\right) .
\end{aligned}
$$

One can extract the following result from the proof of Theorem 1.2 from [4].

Lemma 2.8. Let $H$ be a bipartite graph with $m$ edges and let $\operatorname{gcd}(H)=d \geq 2$. Then, there is $n_{0}=n_{0}(H)$ such that if $G$ is a graph of order $n \geq n_{0}$ with $\phi_{H}(G)=\phi_{H}(n)$ then the following holds:

(1) Let $d_{1}, \cdots, d_{n}$ be the degree sequence of $G$, then

$$
\phi_{H}(G)=\frac{1}{2} \sum_{i=1}^{n} d_{i}-(m-1)\left\lfloor\frac{1}{2 m} \sum_{i=1}^{n}\left\lfloor\frac{d_{i}}{d}\right\rfloor d\right\rfloor .
$$

(2) Let $n=q d+r$ with $0 \leq r \leq d-1$ and $d_{i}=q_{i} d+r_{i}$ with $0 \leq r_{i} \leq d-1$. Then, for $1 \leq i \leq n$ exactly one of the following holds:

(a) $d_{i}=q d-1$;

(b) $i \in C_{1}=\left\{i \in[n] \mid r_{i}=d-1\right.$ and $\left.d_{i}<q d-1\right\}$;

(c) $i \in C_{2}=\left\{i \in[n] \mid d_{i}=n-1\right\}$ if $n-1 \neq R$ and 
$C_{2}=\varnothing$ otherwise.

Furthermore, $\left|C_{1}\right| \leq \frac{2 m}{d}-1$ and $\left|C_{2}\right| \leq 2 m-1$.

In the following we briefly sketch the proof of Lemma 2.8 by giving the argument form [4]. We refrain from doing all the calculations.

Sketch of the proof of Lemma 2.8. Let $\gamma\left(C_{4}\right)$ and $N_{0}$ be given by Lemma 2.7. Assume that $\gamma$ is sufficiently small and that $n_{0} \geq N_{0}$ is sufficiently large to satisfy all the inequalities we will encounter. Let $n \geq n_{0}$ and let $G$ be any graph of order $n$ with $\phi_{C_{4}}(G)=\phi_{C_{4}}(n)$.

Let $G_{n}=G$. Repeat the following at most $\lfloor n / \log n\rfloor$ times: If the current graph $G_{i}$ has a vertex $x_{i}$ of degree at most $(1-\gamma / 2) i$, let $G_{i-1}=G_{i}-x_{i}$ and decrease $i$ by 1 .

Suppose we stopped after $s$ repetitions. Then, either $\delta\left(G_{n-s}\right) \geq(1-\gamma / 2)(n-s)$ or $s=\lfloor n / \log n\rfloor$. Let us show that the later cannot happen. Otherwise, we have

$$
e(G) \leq\left(\begin{array}{c}
n-s \\
2
\end{array}\right)+\left(1-\frac{\gamma}{2}\right) \sum_{i=n-s+1}^{n} i<\left(\begin{array}{l}
n \\
2
\end{array}\right)-\frac{\gamma n^{2}}{4 \log n} .
$$

Let $t$ satisfy $K_{t, t} \supset H$. Using the fact that $\operatorname{ex}\left(n, K_{t, t}\right)=O\left(n^{2-1 / t}\right),(2.1)$ and (2.8) we obtain

$$
\begin{aligned}
\phi_{H}(G) & \leq \frac{1}{m}\left(\left(\begin{array}{l}
n \\
2
\end{array}\right)-\frac{\gamma}{4} \frac{n^{2}}{\log n}\right)+\frac{m-1}{m} c n^{2-1 / t} \\
& <\frac{1}{m}\left(\begin{array}{l}
n \\
2
\end{array}\right) \leq \phi_{H}\left(K_{n}\right),
\end{aligned}
$$

which contradicts our assumption on $G$. Therefore, $s<\lfloor n / \log n\rfloor$ and we have $\delta\left(G_{n-s}\right) \geq(1-\gamma / 2)(n-s)$.

Let $\alpha=2 \gamma$, We will have another pass over the vertices $x_{n}, \cdots, x_{n-s+1}$, each time decomposing the edges incident to $x_{i}$ by $H$-subgraphs and single edges. It will be the case that each time we remove the edges incident to the current vertex $x_{i}$, the degree of any other vertex drops by at most $3 h^{4}$, where $h=v(H)$. Here is a formal description. Initially, let $G_{n}^{\prime}=G$ and $i=n$. If in the current graph $G_{i^{\prime}}$ we have $\operatorname{deg}_{G_{i^{\prime}}}\left(x_{i}\right) \leq \alpha n$, then we remove all $G_{i^{\prime}}$-edges incident to $x_{i}$ as single edges and let $G_{i-1}^{\prime}=G_{i^{\prime}}-x_{i}$.

Suppose that $\operatorname{deg}_{G_{i^{\prime}}}\left(x_{i}\right)>\alpha n$. Then, the set

$$
X_{i}=\left\{y \in V\left(G_{n-s}\right) \mid x_{i} y \in E\left(G_{i^{\prime}}\right)\right\},
$$

has at least $\alpha n-s+1$ vertices. The minimum degree of $G\left[X_{i}\right]$ is

$$
\delta\left(G\left[X_{i}\right]\right) \geq\left|X_{i}\right|-s-\frac{\gamma n}{2}-s \times 3 h^{4} \geq \frac{2}{3}\left|X_{i}\right| .
$$

Let $y \in V(H), A=\Gamma_{H}(y)$ and $a=|A|$. Another result from [4] (Lemma 3.1) states that there is a constant $C$, such that, all but at most $C$ vertices of $G\left[X_{i}\right]$ can be covered by edge disjoint copies of $H-y$ each of them having vertex disjoint sets $A$. Therefore, all but at most $C$ edges between $x_{i}$ and $X_{i}$ can be decomposed into copies of $H$. All other edges incident to $x_{i}$ are removed as single edges. Let $G_{i-1}^{\prime}$ consist of the remaining edges of $G_{i^{\prime}}-x_{i}$ (that is, those edges that do not belong to an $H$-subgraph of the above $x_{i}$ decomposition). This finishes the description of the case $\operatorname{deg}_{G_{i^{\prime}}}\left(x_{i}\right)>\alpha$.

Consider the sets $S=\left\{x_{n}, \cdots, x_{n-s+1}\right\}$, $S_{1}=\left\{x_{i} \in S \mid \operatorname{deg}_{G_{i^{\prime}}}\left(x_{i}\right) \leq \alpha n\right\}$, and $S_{2}=S / S_{1}$. Let their sizes be $s, s_{1}$, and $s_{2}$ respectively, so $s=s_{1}+s_{2}$.

Let $F$ be the graph with vertex set $V\left(G_{n-s}\right) \cup S_{2}$, consisting of the edges coming from the removed $H$ subgraphs when we processed the vertices in $S_{2}$. We have

$$
\phi_{H}(G) \leq \phi_{H}\left(G_{n-s}^{\prime}\right)+\frac{e(F)}{m}+s_{1} \alpha n+s_{2} C+\left(\begin{array}{l}
s \\
2
\end{array}\right) .
$$

We know that $\phi_{H}\left(G_{n-s}^{\prime}\right)=e\left(G_{n-s}^{\prime}\right)-p_{H}\left(G_{n-s}^{\prime}(m-1)\right)$, furthermore, $\delta\left(G_{n-s}^{\prime}\right) \geq(1-\gamma)(n-s)$. Thus, $p_{H}\left(G_{n-s}^{\prime}\right)$ can be estimated using Lemma 2.7 .

If (2.6) holds, some calculations show that there exits a graph $G^{*}$ such that $\phi_{H}(G)<\phi_{H}\left(G^{*}\right)$, which contradicts the optimality go $G$.

Therefore, (2.5) must hold. It follows that $p_{H}(G)$ and thus $\phi_{H}(G)$, depends only on the degree sequence $d_{1}, \cdots, d_{n}$ of $G$. Namely, the packing number

$\ell=p_{H}(G)$ equals $\left\lfloor\frac{1}{2 m} \sum_{i=1}^{n} r_{i}\right\rfloor$, where $r_{i}=d\left\lfloor d_{i} / d\right\rfloor$ is the largest multiple of $d$ not exceeding $d_{i}$.

Therefore,

$$
\phi_{H}(G)=\frac{1}{2} \sum_{i=1}^{n} d_{i}-(m-1)\left\lfloor\frac{1}{2 m} \sum_{i=1}^{n}\left\lfloor\frac{d_{i}}{d}\right\rfloor d\right\rfloor,
$$

where $d_{1}, \cdots, d_{n}$ is the degree sequence of $G$.

To conclude the proof we need to estimate the values that the degrees of $G$ can attain. To do that we need to prove an upper bound on $\phi_{H}(G)$ by estimating $\phi_{\max }$, the maximum of

$$
\phi\left(d_{1}, \cdots, d_{n}\right)=\frac{1}{2} \sum_{i=1}^{n} d_{i}-(m-1)\left\lfloor\frac{1}{2 m} \sum_{i=1}^{n}\left\lfloor\frac{d_{i}}{d}\right\rfloor d\right\rfloor,
$$

over all (not necessarily graphical) sequences $d_{1}, \cdots, d_{n}$ of integers with $0 \leq d_{i} \leq n-1$.

Let $d_{1}, \cdots, d_{n}$ be an optimal sequence attaining the value $\phi_{\max }$. For $i=1, \cdots, n$ let $d_{i}=q_{i} d+r_{i}$ with $0 \leq r_{i} \leq d-1$. Then, $\ell=\left\lfloor\frac{\left(q_{1}+\cdots+q_{n}\right) d}{2 m}\right\rfloor$. 
Let $n=q d+r$ with $0 \leq r \leq d-1$ and $q=\lfloor n / d\rfloor$. Define $R=q d-1$ to be the maximum integer which is at most $n-1$ and is congruent to $d-1$ modulo $d$. Let $C_{1}=\left\{i \in[n] \mid r_{i}=d-1\right.$ and $\left.d_{i}<R\right\}$ and $C_{2}=\left\{i \in[n] \mid d_{i}=n-1\right\} \quad$ if $\quad n-1 \neq R \quad$ and $\quad C_{2}=\varnothing$ otherwise.

Since $d_{1}, \cdots, d_{n}$ is an optimal sequence, we have that if $r_{i} \neq d-1$ then $d_{i}=n-1$ for all $i \in[n]$. To conclude the proof it remains to show that $\left|C_{1}\right| \leq \frac{2 m}{d}-1$ and $\left|C_{2}\right| \leq 2 m-1$. Suppose first that $\left|C_{1}\right| \geq \frac{2 m}{d}=: t$. Consider the new sequence of integers

$$
d_{i}^{*}= \begin{cases}d_{i}+d, & \text { if } i \in C_{1}, \\ d_{i}, & \text { if } i \notin C_{1} .\end{cases}
$$

Then, $\ell^{*}=\ell+1$ and $\phi^{*}=\phi_{\max }+1$ which contradicts our assumption on $\phi_{\max }$.

Now suppose that $\left|C_{2}\right| \geq 2 m$ and consider the new sequence of integers $d_{1}^{*}, \cdots, d_{n}^{*}$ obtained from $d_{1}, \cdots, d_{n}$ by replacing $2 m$ values of $n-1$ by $R$. Then, $\ell^{*}=\ell-d$ and $\phi^{*} \geq \phi_{\max }+m-d>\phi_{\max }$, which contradicts our assumption on $\phi_{\max }$ and the proof is concluded.

We now have all the tools needed to prove Theorem 1.4.

Proof of Theorem 1.4. Let $n_{0}$ be given by Lemma 2.8. Let $G$ be a graph of order $n \geq n_{0}$ with $\phi_{C_{4}}(G)=\phi_{C_{4}}(n)$ and degree sequence $d_{1}, \cdots, d_{n}$. For $i=1, \cdots, n$ let $d_{i}=2 q_{i}+r_{i}$ with $0 \leq r_{i} \leq 1$. Let, $R=2(\lfloor n / 2\rfloor-1)+1$ and let the sets $C_{1}$ and $C_{2}$ be as in Lemma 2.8 .

Let $n=2 q+r$ with $0 \leq r \leq 1$ and $q=\lfloor n / 2\rfloor$. From (2.7) we obtain

$$
\begin{aligned}
& \phi_{C_{4}}(n) \\
& =n(q-1)+\frac{n}{2}+\frac{1}{2}\left|C_{2}\right| r-\sum_{i \in C_{1}}\left(q-1-q_{i}\right) \\
& -3\left\lfloor\frac{1}{4} n(q-1)+\frac{1}{4}\left|C_{2}\right|-\frac{1}{4} \sum_{i \in C_{1}}\left(q-1-q_{i}\right)\right\rfloor
\end{aligned}
$$

In what follows let $\alpha=\left|C_{2}\right|$ and $\beta=\sum_{i \in C_{1}}\left(q-1-q_{i}\right)$.

We consider first the case when $n$ is even. Then $C_{2}=\varnothing$ and we have

$$
\begin{aligned}
\phi_{C_{4}}(n) & =n(q-1)+\frac{n}{2}-\beta-3\left\lfloor\frac{1}{4} n(q-1)-\frac{\beta}{4}\right\rfloor \\
& =n(q-1)+\frac{n}{2}-\frac{3 q(q-1)}{2}-\beta-3\left\lfloor-\frac{\beta}{4}\right\rfloor
\end{aligned}
$$

Claim 1. Let $d_{1}, \cdots, d_{n}$ be the degree sequence of a graph. Then,

$$
-\beta-3\left\lfloor-\frac{\beta}{4}\right\rfloor \leq 1
$$

Proof. Routine calculations show that for $\beta \neq 1$ we have $-\beta-3\left\lfloor-\frac{\beta}{4}\right\rfloor \leq 1$. Suppose $\beta=1$. Then $C_{1}$ has exactly one element, thus the sequence $\left(d_{i}\right)_{i=1, \cdots, n}$ has exactly one element equal to $n-3$ and all the others equal to $n-1$. But this is not a degree sequence of a graph since condition (2.3) of Theorem 2.6 does not hold for $k=n-2$.

Therefore, using the estimate of Claim 1 in (2.13) it follows that

$$
\phi_{C_{4}}(n) \leq \frac{n^{2}}{8}+\frac{n}{4}+1 .
$$

To prove the lower bound consider the graph $L_{5}$ obtained from $K_{n}$ after the deletion of the edges of a $C_{5}$. Using (1.1) and (2.5) we show that

$$
\phi_{C_{4}}\left(L_{5}\right)=\frac{n^{2}}{8}+\frac{n}{4}+1 .
$$

We now consider the case when $n$ is an odd number.

Case 1: Let $n=8 t+1$ and $q=4 t$.

From (2.12) we obtain

$$
\begin{aligned}
\phi_{C_{4}}(n)= & \frac{n}{2}(n-3)+\frac{n}{2}-3\left(8 t^{2}-t\right) \\
& +\frac{1}{2} \alpha-\beta-3\left\lfloor\frac{\alpha-\beta-1}{4}\right\rfloor
\end{aligned}
$$

Claim 2. Let $d_{1}, \cdots, d_{n}$. be the degree sequence of a graph. Then,

$$
\frac{1}{2} \alpha-\beta-3\left\lfloor\frac{\alpha-\beta-1}{4}\right\rfloor \leq \frac{5}{2} .
$$

Proof. Routine calculations show that the result follows if $\alpha \neq 0$ or $\beta \neq 0$. If $\alpha=0$ and $\beta=0$ then $d_{i}=n-2$ for all $1 \leq i \leq n$. This is not a degree sequence of a graph since $\sum_{i=1}^{n} d_{i}$ is not even.

Therefore, using the estimate of Claim 2 in (2.14) we prove that

$$
\phi_{C_{4}}(n) \leq \frac{n^{2}}{8}+\frac{n}{8}+\frac{14}{8} .
$$

As for the lower bound consider the graph $L^{*}$ with all vertices of degree $n-2$ except one of degree $n-3$. Using (1.1) and (2.5) we show that

$$
\phi_{C_{4}}\left(L^{*}\right)=\frac{n^{2}}{8}+\frac{n}{8}+\frac{14}{8} .
$$

Case 2: Let $n=8 t+3$ and $q=4 t+1$.

From (2.12) we obtain 


$$
\begin{aligned}
\phi_{C_{4}}(n)= & \frac{n}{2}(n-3)+\frac{n}{2}-3\left(8 t^{2}+3 t\right) \\
& +\frac{1}{2} \alpha-\beta-3\left\lfloor\frac{\alpha-\beta}{4}\right\rfloor .
\end{aligned}
$$

Claim 3. Let $d_{1}, \cdots, d_{n}$. be the degree sequence of a graph. Then,

$$
\frac{1}{2} \alpha-\beta-3\left\lfloor\frac{\alpha-\beta}{4}\right\rfloor \leq \frac{3}{2} .
$$

Proof. It follows from routine calculations for all values of $\alpha$ and $\beta$ except when $\alpha=0$ and $\beta=1$. Suppose that $\alpha=0$ and $\beta=1$. Then $C_{2}=\varnothing$ and $C_{1}$ has exactly one element, thus the sequence $\left(d_{i}\right)_{i=1, \cdots, n}$ has exactly one element equal to $n-2$ and all the others equal to $n-1$. But this is not a degree sequence of a graph since $\sum d_{i}$ is not even.

Therefore, using the estimate of Claim 3 in (2.15) we prove that

$$
\phi_{C_{4}}(n) \leq \frac{n^{2}}{8}+\frac{n}{8}+\frac{3}{2} .
$$

As for the lower bound consider the graph $L$ with degree sequence $d_{1}=d_{2}=n-4, d_{3}=\cdots=d_{n-1}=n-2$ and $d_{n}=n-1$ (the existence of $L$ can be proved directly or by Erdös-Gallai theorem, Theorem 2.6). Using (1.1) and (2.5) we show that

$$
\phi_{C_{4}}(L)=\frac{n^{2}}{8}+\frac{n}{8}+\frac{3}{2} .
$$

Case 3: Let $n=8 t+5$ and $q=4 t+2$.

From (2.12) we obtain

$$
\begin{aligned}
\phi_{C_{4}}(n)= & \frac{n}{2}(n-3)+\frac{n}{2}-3\left(8 t^{2}+7 t\right) \\
& +\frac{1}{2} \alpha-\beta-3\left\lfloor\frac{\alpha-\beta+5}{4}\right\rfloor .
\end{aligned}
$$

Claim 4. Let $d_{1}, \cdots, d_{n}$. be the degree sequence of a graph. Then,

$$
\frac{1}{2} \alpha-\beta-3\left\lfloor\frac{\alpha-\beta+5}{4}\right\rfloor \leq-\frac{5}{2} .
$$

Proof. Routine calculations show that

$\alpha / 2-\beta-3\left\lfloor\frac{\alpha-\beta+5}{4}\right\rfloor \leq-5 / 2$ for all values of $\alpha$ and $\beta$ except for $\alpha=2$ and $\beta=0$ or $\alpha=0$ and $\beta=2$.

Suppose first that $\alpha=2$ and $\beta=0$. Then the sequence $\left(d_{i}\right)_{i=1, \ldots, n}$ has two elements equal to $n-1$ and all the others equal to $n-2$. This is not a degree sequence of a graph since $\sum_{i=1}^{n} d_{i}$ is not even.

Suppose now that $\alpha=0$ and $\beta=2$. If $\left|C_{1}\right|=2$ then the sequence has two elements equal to $n-4$ and all the others equal to $n-2$ and this is not a degree sequence of a graph since $\sum d_{i}$ is not even. Finally, if $\left|C_{1}\right|=1$ then we have one element equal to $n-6$ and all the others equal to $n-2$. Again, this is not a degree sequence of a graph since $\sum d_{i}$ is not even.

Therefore, using the estimate of Claim 4 in (2.16) we prove that

$$
\phi_{C_{4}}(n) \leq \frac{n^{2}}{8}+\frac{n}{8}+\frac{10}{8} .
$$

As for the lower bound consider the graph $K_{n}-I$ obtained from $K_{n}$ by deleting the edges of a maximum matching. Using (1.1) and (2.5) we show that

$$
\phi_{C_{4}}\left(K_{n}-I\right)=\frac{n^{2}}{8}+\frac{n}{8}+\frac{10}{8} \text {. }
$$

Case 4: Let $n=8 t+7$ and $q=4 t+3$.

From (2.12) we obtain

$$
\begin{aligned}
\phi_{C_{4}}(n)= & \frac{n}{2}(n-3)+\frac{n}{2}-3\left(8 t^{2}+11 t\right) \\
& +\frac{1}{2} \alpha-\beta-3\left\lfloor\frac{\alpha-\beta+14}{4}\right\rfloor .
\end{aligned}
$$

Claim 5. Let $d_{1}, \cdots, d_{n}$. be the degree sequence of a graph. Then,

$$
\frac{1}{2} \alpha-\beta-3\left\lfloor\frac{\alpha-\beta+14}{4}\right\rfloor \leq-\frac{17}{2} .
$$

Proof. It follows directly from simple calculations. $\square$

Therefore, using the estimate of Claim 5 in (2.17) we prove that

$$
\phi_{C_{4}}(n) \leq \frac{n^{2}}{8}+\frac{n}{8}+2
$$

Furthermore, using (1.1) and (2.5) we have

$$
\phi_{C_{4}}\left(K_{n}-I\right)=\frac{n^{2}}{8}+\frac{n}{8}+2,
$$

so the equality follows and the proof is now complete. $\square$

\section{Acknowledgements}

The author would like to thank Oleg Pikhurko for helpful comments and discussions. The author acknowledges the support from FCT-Fundação para a Ciência e a Tecnologia (Portugal), through Projects PTDC/MAT/113207/ 2009 and PEst-OE/MAT/UI0297/2011 (CMA).

\section{REFERENCES}

[1] B. Bollobás, "Modern Graph Theory," Springer-Verlag, New York, 1998. doi:10.1007/978-1-4612-0619-4

[2] P. Erdös, A. W. Goodman and L. Pósa, "The Representation of a Graph by Set Intersections," Canadian Journal 
of Mathematics, Vol. 18, No. 1, 1966, pp. 106-112. doi:10.4153/CJM-1966-014-3

[3] B. Bollobás, "On Complete Subgraphs of Different Orders," Mathematical Proceedings of the Cambridge Philosophical Society, Vol. 79, No. 1, 1976, pp. 19-24. doi:10.1017/S0305004100052063

[4] O. Pikhurko and T. Sousa, "Minimum H-Decompositions of Graphs," Journal of Combinatorial Theory Series B, Vol. 97, No. 6, 2007, pp. 1041-1055. doi:10.1016/j.jctb.2007.03.002

[5] T. Sousa, "Decompositions of Graphs into a Given CliqueExtension," ARS. Combinatoria, Vol. 100, 2011, pp. 465472.

[6] T. Sousa, "Decompositions of Graphs into 5-Cycles and Other Small Graphs," Electronic Journal of Combinatorics, Vol. 12, 2005, 7p.

[7] T. Sousa, "Decompositions of Graphs into Cycles of Length Seven and Single Edges," ARS. Combinatoria, to appear.
[8] L. Özkahya and Y. Person, "Minimum H-Decompositions of Graphs: Edge-Critical Case," Journal of Combinatorial Theory Series B, Vol. 102, No. 102, 2012, pp. 715-725. doi:10.1016/j.jctb.2011.10.004

[9] P. Allen, J. Böttcher and Y. Person, "An Improved Error Term for Minimum H-Decompositions of Graphs," arXiv: 1109.2571v1, 2011.

[10] P. Erdös and T. Gallai, "Graphs with Prescribed Degree of Vertices," Matematikai Lapok, Vol. 11, 1960, pp. 264274.

[11] N. Alon, Y. Caro and R. Yuster, "Packing and Covering Dense Graphs," Journal of Combinatorial Designs, Vol. 6, No. 6, 1998, pp. 451-472. doi:10.1002/(SICI)1520-6610(1998)6:6<451::AID-JCD6 3.0.CO;2-E

[12] T. Gustavsson, "Decompositions of Large Graphs and Digraphs with High Minimum Degree," Ph.D. Thesis, University of Stockholm, Stockholm, 1991. 